Research Article

\title{
Power Indices in the Context of Social Learning Behaviour in Social Networks
}

\author{
Ruili Shi $\mathbb{D}^{1,2}$ Chunxiang Guo $\mathbb{D}^{1},{ }^{1}$ and Xin Gu $\mathbb{D}^{1}$ \\ ${ }^{1}$ Business School, Sichuan University, Chengdu 610065, China \\ ${ }^{2}$ Chongqing Business Vocational College, Chongqing 401331, China \\ Correspondence should be addressed to Chunxiang Guo; guochunxiang@scu.edu.cn
}

Received 30 March 2019; Revised 30 May 2019; Accepted 2 June 2019; Published 25 June 2019

Academic Editor: Marko Robnik

Copyright (c) 2019 Ruili Shi et al. This is an open access article distributed under the Creative Commons Attribution License, which permits unrestricted use, distribution, and reproduction in any medium, provided the original work is properly cited.

This paper puts forward the concept of integrated power, synthetically measures the voters' ability to influence the results of decision-making by influencing others through social learning, considering the interactions between decision-makers in social networks, and offers a method for measuring integrated power. Based on the theory and model of social learning, we analyze the influence of social learning on the voting process and power indices from the perspective of individuals' professional level, position within the social network structure, relationship closeness, and learning efficiency. A measurement model of integrated power is constructed, and the variation in integrated power compared with that of the Banzhaf index is analyzed by numerical simulation. The results show that when the individual's professional level is higher and closeness with neighboring decision-makers is greater, then the integrated power index is higher. An individual's integrated power index may decrease when he/she changes from an isolated node to a nonisolated node, and then his/her integrated power will increase with the increases of neighbor nodes. Social learning efficiency can promote the integrated power of individuals with lower social impact and relationship closeness, but it is not beneficial for the core and influential members of the social network.

\section{Introduction}

A core issue in group decision-making problems, such as voting games, is how to construct a preference sequence based on individuals' preferences. The power distribution of voters is the basis for the prioritization and selection of the best-or at least, a satisfactory-scheme, which is related to the credibility of the voting results. Therefore, power analysis became one of the main issues in the analysis of a voting situation.

Voting power, that is, a voter's capacity to influence the voting procedure, can reflect the fairness and rationality of the voting rules and is always the focus of scholarly study. Several remarkable power indices, such as the Shapley-Shubik index [1] and the Banzhaf index [2], have been introduced in the literature on simple games and have been applied to many practical problems such as decision conflict resolution, responsibility sharing, benefit distribution, equity allocation, and so on $[3,4]$. The classical power indices measure voting power under the assumption that voters cast their votes independently and that each voter is equally likely to vote "yes" or "no". These measures are not supported empirically and fail to account for the preferences of and interactions between voters. A standard power index is therefore considered as a priori power related only to the voting rules $[5,6]$ and can best be described as a constitutional voting power, that is, the power that is inherent in the formal rules of the voting game only and that does not consider the behaviour in an actual voting system [7]. These models of voting power do not seem to capture actual a posteriori voting power, where preferences and interdependence may exist. Therefore, many later literatures give up the assumptions of independence and random voting and propose corresponding voting power indices, such as spatial power indices [8,9], empirical power indices [5], behavioural power indices [7], causal dependence power indices $[10,11]$, probabilistic power indices for voting with abstention [12], and other modified indices [13, 14]. These mentioned power indices consider voters' preferences 
and behaviour in actual voting systems; however, they are powerless to reflect managerial phenomena caused by complex social interactions within organizations.

Group interactions are indeed inseparably linked with our increasingly interconnected world, and interactions among human societies are more complex [15-17]. As complex network structures are more representative for human societies, group interactions on complex network had been widely studied and present how social diversity affects human behaviour (for a review, see [15]). Human experiments of cooperation have also suggested that social relationship network structure is an important factor of human behaviours [16]. In group decision-making process, the structured relationships between voters may also influence their behaviours and judgments $[18,19]$. Thus, later literatures have partially considered heterogeneous voters and their social structure when measuring power indices. The most representative of these models are opinion leadership models, which are based on the theory of the two-step flow of communication [20]. The properties and axiomatization of opinion formation under a leader-follower power dynamic have been provided [21-23], but voter heterogeneity in such models takes a simple form (leader and follower). Such relationships are not general, as relations among members are usually equal, such as in citizens' elections, and do not exist an "institutional power." In addition, the voting process is usually a process of information exchange and learning among members that is based on social relationships [24].

Relationships among members can be described by social networks, which provide a new way to study the behaviour and influence of relationships among individuals and organizations in society. Thus, the theory and methods of social networks have been introduced into problems of group decision-making [25-28]. Network analysts implicitly assume that power is related to network centrality [29-33], but they do not consider the voting rule that is in effect when analyzing group decisions with social networks.

This paper focuses on a power analysis based on social networks. The problem is complex: on the one hand, a social network enhances the interaction between decision-makers, and the network effect may enlarge or reduce the influence of decision-makers on the results; on the other hand, in the social networks, people can observe and obtain others' behavioural information more conveniently and update or strengthen their own judgments, which increase the variability in individual preference. These characteristics require that power indices not only reflect the direct influence that voters have on results but also consider the indirect influence on voting result through social interactions.

In this paper, we analyze the process of group interactions informed by social learning theory [34] and construct a power measurement model based on social networks. Social learning theory has been widely used in the fields of sociology, psychology, pedagogy, and management science [24, $35,36]$ and holds that individual's behaviours are deeply influenced by the social environment and that individuals will determine or change their views and behaviours according to the observed behaviours [34-36]. The group interactions during voting process can be regarded as a process of behavioural observation and social learning. Therefore, we study the influence group interactions have on voting based on social learning theory and analyze the influence of these interactions on power indices.

The paper is organized as follows: Section 2 introduces the concepts of power indices, social network, and social learning theory; Section 3 describes the integrated power measure model informed by social learning theory based on social networks; next, in Section 4, we present a numerical example to analyze the influence of social learning on power indices; and finally, Section 5 provides a brief summary.

\section{Preliminaries}

This section presents a brief review of the main concepts used in this research. We introduce classic power indices briefly and explain the index measure method that our model extends. Since our model is based on social networks, this theory is briefly reviewed. Social learning theory is another key concept used in this research; therefore, the concepts of social learning and social impact are reviewed briefly, and the phases in social learning modeling are introduced. A brief description of a power indices model based on social learning is also presented at the end of this section.

2.1. Power Indices. Power is a voter's capacity to influence the voting procedure. Several important and now standard power indices such as the Shapley-Shubik index [1] and the Banzhaf power index [2] have been introduced in the literature for simple games. Their axiomatizations have been provided by, among others, Shapley [8] and Owen [9]. These power indices have been modified and extended in various ways $[10-14,37]$.

A group decision-making problem can be described as an ordered binary $(X, v)=[q ; w]$, in which $X=\{1,2,3, \cdots, n\}$ is the set of voting members with weights of $W=\left\{w_{i}\right\}_{1 \times n}$, and $q$ is the winning threshold. The two most well-known power indices for binary voting games (voting between "Yes" and "No") are the Shapley-Shubik index [1] and the Banzhaf index [2]. The difference between these two indices is that the Shapley index calculates the winning probability of an individual based on sorting, while the Banzhaf index calculates the same probability based on combinations of individuals [38]. This paper analyzes the influence of social learning on a power distribution based on Banzhaf index.

Let $v$ be a voting game that consists of $X=\{1,2,3, \cdots, n\}$ voters with two alternatives (i.e., vote "Yes" or "No") and satisfies $v\left(C_{Y}\right)=1$ if $w\left(C_{Y}\right) \geq q, v\left(C_{N}\right)=0$ if $w\left(C_{Y}\right)<q$, where $C_{l}$ is the set of voters who choose alternative $l(l=$ $Y, N)$, and $w\left(C_{Y}\right)$ is the voting weight of voters in $C_{l}$. In this case, $C=\left(C_{Y}, C_{N}\right)$ is called an arrangement of the players among the two alternatives. The function $v: 2^{X} \longrightarrow\{0,1\}$ is monotonic $\left(C_{Y} \subseteq S_{Y} \Longrightarrow v\left(C_{Y}\right) \leq v\left(S_{Y}\right)\right)$ and satisfies $v(\phi)=0, v(X)=1$. Then, the Banzhaf index of voter $i$ is

$$
B_{i}(v)=\frac{1}{2^{n-1}} \times \sum_{C: i \in C_{Y}}\left(v\left(C_{Y}\right)-v\left(C_{Y} \backslash\{i\}\right)\right),
$$


and the relative power index is

$$
B_{i}^{\prime}(v)=\frac{B_{i}(v)}{\sum_{i} B_{i}(v)} .
$$

2.2. Social Network Analysis. Due to the overwhelming evidence indicating that complex network promotes the description of the complex relationship in human society [15], social network analysis (SNA) - an important branch of complex network science-has become one of the most popular methodologies for investigating social relationships among group members. It provides an effective way to analyze interconnection structures that more representative for human societies [15-17] and has been introduced into analysis of group decision-making [26-28].

Social networks have been modeled as a graph of relationships among individuals. In a voting group, the social relations between members can be described as a social network $G=(X, \kappa, E, M)$ (shown in Figure 1) with voters as nodes, where $X=\{1,2,3, \cdots, n\}$ is the set of voters, $\kappa=\left\{k_{i}\right\}$, where $k_{i}$ is the weight of node $i$, representing its attributes (e.g., level of expertise, level of professional knowledge, etc.), $E=\left\{e_{i j}\right\}$ is the set of edges representing the social relationship of members, and $M=\left\{m_{i j}\right\}$ is the set of $e_{i j}$ 's weights representing the closeness of $i, j$.

Previous research shows that the structure and characteristics of a social network determine the behaviours and opinions of humans $[15,16]$. To measure the importance or influence of decision-makers in a social network, the concept of power was introduced into network analysis [27, 28]. Network analysis assume that power is related to node criticality, such as network centrality $[30,31]$ or connectivity [39, 40]. More recently, new ideas have been proposed. Morone and Makse [32] mapped the problem onto optimal percolation in random networks to identify the minimal set of influencers and introduced the collective-influence algorithm. To adapt the Banzhaf index and discover the critical node in a network, Asif et al. [41] proposed the combined Banzhaf \& diversity index. Such network centrality measures incorporate network structures but do not consider voting rules.

2.3. Social Learning Theory. Power indices in the context of social network should include not only the direct impact of the individual on the outcome of the decision (direct power) but also the influence gained through influence on other individuals (indirect power). In a group decision-making problem, the group interaction process is essentially the process of obtaining information through empirical search or behavioural observation and learning [34]. People often believe that they can obtain useful information from others' actions and use that information to improve the correctness of their own decisions; i.e., they can experience social learning.

Social learning theory has been widely used in sociology, psychology, and behavioural economics $[35,36]$. The theory of social learning shows that social learning efficiency is

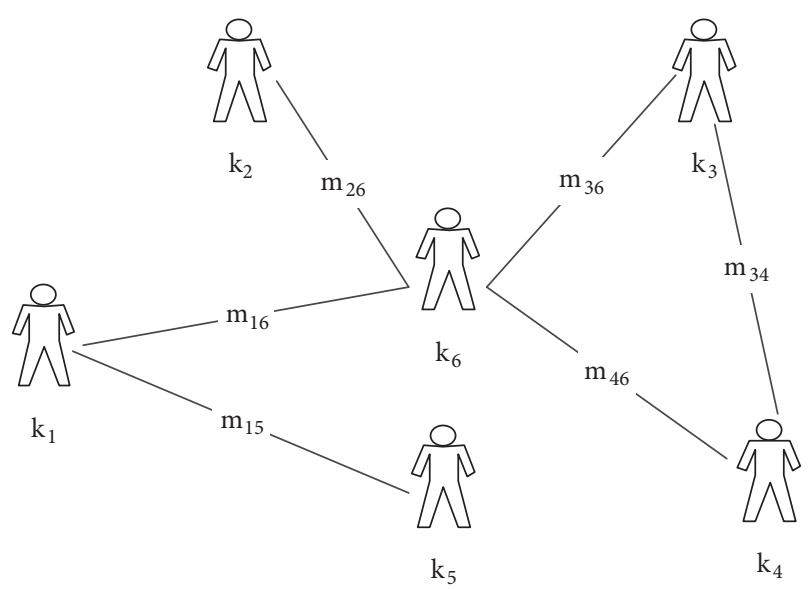

FIGURE 1: Social network of voting groups.

related to the attributes of the individual, that is, their social influence:

$$
p\left(O(i) \mid\left(s(i), S_{i}\right)\right)=<s(i), S_{i}, I_{i}>,
$$

where $O(i)$ is the postbehaviour of individual $i ; s(i)$ is the personal information (behaviour or opinion) of $i$; $S_{i}$ is the behaviour of other individuals observed by individual $i$; and $I_{i}$ is the social impact on individual $i$.

Social impact is a common social phenomenon that refers to the effect on people's behaviours of the surrounding social environment. According to the social impact theory, which was formulated by Latané (Latané 1981), such impact depends on three factors [42]: (1) the social distance of sources from the subject, which could be spatial proximity or closeness in space, time or an abstraction of the personal relationship; (2) the sources' strength or power of persuasion/support; and (3) the number of sources. Thus, the total influence that individual $i$ experiences from the society is

$$
\begin{aligned}
I_{i}= & {\left[\sum_{j=1}^{n} \xi_{j} \cdot \gamma_{i j}(1-\sigma(i) \sigma(j))\right] } \\
& -\left[\sum_{j=1}^{n} \zeta_{j} \bullet \gamma_{i j}(1+\sigma(i) \sigma(j))\right],
\end{aligned}
$$

where $\xi_{i}$ is $i$ 's persuasiveness and $\zeta_{i}$ is $i$ 's supportiveness, describing the agent's capability to convince another agent to change or persist in its opinion, respectively; $\gamma_{i j}$ is the closeness between $i, j$; and $\sigma(i)= \pm 1$ respects the binary opinions of individuals. When the pressure to change one's opinion is greater than the pressure to maintain one's point of view, that is, when $I_{i}>0$, then the opinion changes.

This paper aims to analyze the influence of individual interactions on voting processes and voting power. It is assumed that the interaction between individuals in group decision-making is essentially a process for individuals to renew their opinions through social learning. The interaction process in group decision-making can be described as in Figure 2. 


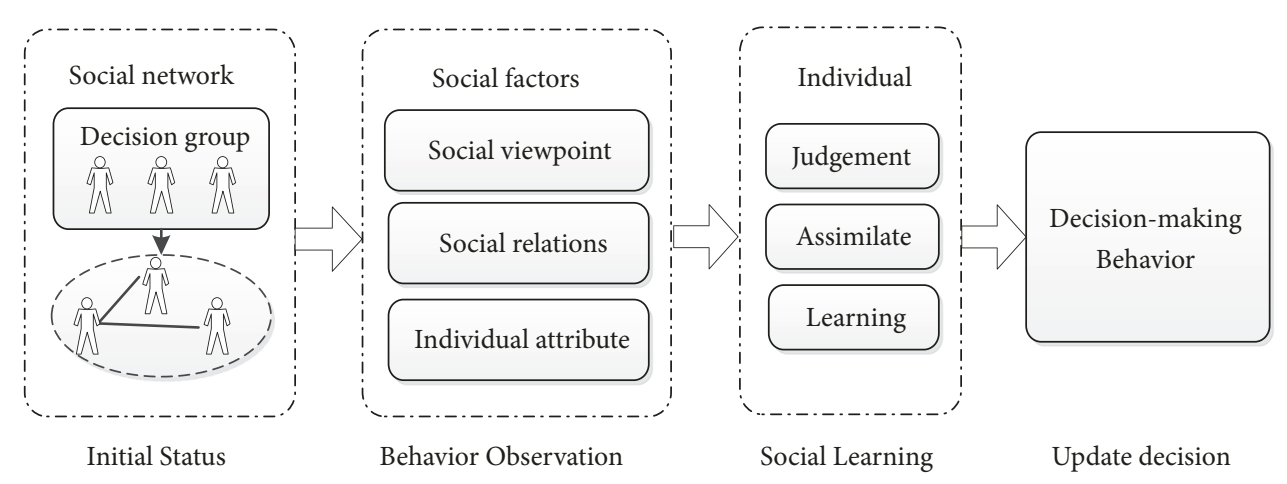

FIGURE 2: The social learning process of voting groups based on social networks.

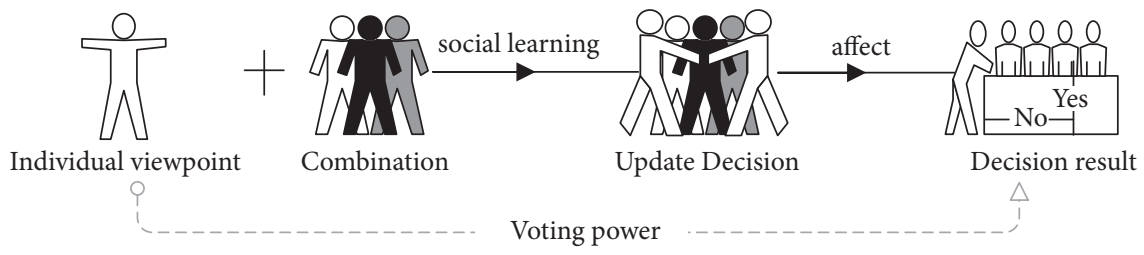

FIGURE 3: The influence of social learning on the voting process.

Based on social learning theory, the process of decisionmakers influencing voting results can be described as in Figure 3.

\section{Proposed Model}

Our proposed model of power indices is based on social learning theory. The interactive voting game is essentially a process of behavioural observation learning based on a social network.

3.1. Social Learning Model. The model consists of $X=$ $\{1,2,3, \cdots, n\}$ voters. The voters share their opinions $s(i)=$ $\pm 1(i=1,2, \cdots, n)$ based on social network $G=(X, \kappa, E, M)$. Let $C^{0}=\left(C_{Y}{ }^{0}, C_{N}{ }^{0}\right)$ be the initial distribution of group preference, and let $S_{i}=\left\{s(j) \mid j \in \Gamma_{i}\right\}$ be the preference combination observed by individual $i . S_{i}=\left\{s(j) \mid j \in \Gamma_{i}\right\}$ where $\Gamma_{i}$ is the collection of $i$ 's neighbor nodes,

$$
s(i)= \begin{cases}1, & i \in C_{Y}^{0} \\ -1, & i \in C_{N}^{0} .\end{cases}
$$

The total influence that individual $i$ experiences from the society is

$$
\begin{aligned}
I_{i}= & \alpha^{-} \sum_{j} \lambda_{j} \times \gamma_{i j} \times(1-s(i) s(j))-\alpha^{+} \sum_{j} \lambda_{j} \times \gamma_{i j} \\
& \times(1+s(i) s(j))
\end{aligned}
$$

where $\alpha^{+}, \alpha^{-}$are the influence parameters of the same or the different views, respectively; $\alpha^{+}, \alpha^{-} \geq 0, \alpha^{+}>\alpha^{-}$means that the individual is more receptive to the same point of view; $\lambda_{i}$ is the social influence (persuasiveness or supportiveness); and $\gamma_{i j}$ is the closeness between $i, j$.

Social influence can be defined as "change in the belief, attitude, or behaviour of a person that results from the action or presence of another person" [43]. In the original social impact model, the strengths of persuasiveness and supportiveness are assigned to the agents according to a uniform random distribution. However, in the real world, social influence, that is, social power, depends not only on the agent's own professionalism, which is the basis of social power, but also on his/her structural position in the social network. Researches on network science have shown that social power depends on the individual's connection structure in the network [44] and suggested that the wellconnected nodes should be considered influential people $[30,39,40]$. Therefore, social power could be given as follows.

Definition 1 (social power). If in a social network $G=$ $(X, \kappa, E, M), d_{i}$ is the node degree of $i, k_{i}$ is $i$ 's knowledge level, and the network centrality of $i$ is

$$
C_{D}(i)=\frac{d_{i}}{n-1}
$$

then the social power of $i$ is

$$
\lambda_{i}=k_{i}^{\prime} \times C_{D}(i),
$$

where $k_{i}^{\prime}$ is the normalized value of $k_{i}, k_{i}^{\prime}=k_{i} / \max _{i=1}^{n}\left(k_{i}\right)$.

The relationship closeness is another factor in social behaviour learning. In traditional social impact models, the closeness of any pair of agents is usually measured by the distance function. In fact, the closeness degree can be determined by the frequency of communication. 
Definition 2 (relationship closeness). In a social network $G=$ $(X, \kappa, E, M)$, the closeness of any pair of nodes $i, j$ can be assigned as

$$
\gamma_{i j}=\frac{m_{i j}}{\max (M)} .
$$

According to (6), when the pressure of persuasiveness is less than that of supportiveness, that is, $I_{i}<0$, the individual tends to keep his or her original view; if the opposite is true and the pressure of supportiveness is less than that of persuasiveness, the agent tends to change his or her own opinion. Especially when an individual has nonneighbor node, then its social impact $I_{i}=-\infty$.

Based on the analysis above, (3) could be rewritten for the model as

$$
\begin{gathered}
p\left(O(i)=-s(i) \mid\left(s(i), S_{i}\right)\right)=\frac{1}{1+e^{-2\left(I_{i} / b\right)}} \\
p\left(O(i)=s(i) \mid\left(s(i), S_{i}\right)\right)=\frac{1}{1+e^{2\left(I_{i} / b\right)}}
\end{gathered}
$$

where $b$ is a coefficient of social impact, expressing the social learning efficiency of the group, $b>0$.

For individual $i, p\left(O(i)=o \mid\left(s(i), S_{i}\right)\right) \in[0,1]$ and $\sum_{o} p\left(O(i)=o \mid\left(s_{i}, S_{i}\right)\right)=1(o=-1,1)$.

3.2. Power Indices Model. The ability of an individual to influence the outcome changes when social learning takes place. Let $C_{Y} \mid C^{0}$ be a set of decision-makers who vote "Yes" after an opinion interaction when the initial arrangement of the voters is $C^{0}=\left(C_{Y}{ }^{0}, C_{N}{ }^{0}\right)$. If voter $i \in C_{Y}{ }^{0}$, and after the opinion interaction the "Yes" alternative is chosen, and when the initial inclination of voter $i$ is changed from "Yes" to "No," and then after the opinion interaction the outcome of group is "No," that is, $v\left(C_{Y} \mid C^{0}\right)=1$ and $v\left(C_{Y} \mid\left(C_{Y}{ }^{0} \backslash\{i\}, C \cup\{i\}\right)\right)=0$ if $i \in C_{Y}^{0}$, we say that the individual $i$ influences the outcome of voting through both direct voting and influence on others' voters, which is called the integrated power of voters.

Definition 3 (integrated power index). In a group decisionmaking problem $(X, v)=[q ; w]$ with the social network $G=(X, \kappa, E, M)$, let $\mu_{i}\left(C^{0}\right)=p\left(O(i)=1 \mid\left(s_{i}, S_{i}\right)\right)$ be the probability of voting "Yes" after social learning; then, the probability of a combination after interaction is

$$
\left.\pi\left(C_{Y} \mid C^{0}\right)=\prod_{i \in C_{Y} \mid C^{0}} \mu_{i}\left(C^{0}\right) \times \prod_{j \notin C_{Y} \mid C^{0}}\left(1-\mu_{j}\left(C^{0}\right)\right)\right),
$$

Let the set of winning combinations be $V$, then $i$ 's integrated power index is

$$
\begin{aligned}
& R_{i}(v)=\frac{1}{2^{n-1}} \sum_{C^{0}: i \in C_{Y}^{0}}\left(\sum_{C_{Y} \mid C^{0} \in V} \pi\left(C_{Y} \mid C^{0}\right)\right. \\
& \left.-\sum_{C_{Y} \mid\left(C_{Y}{ }^{0} \backslash\{i\}, C \cup\{i\}\right) \in V} \pi\left(C_{Y} \mid\left(C_{Y}{ }^{0} \backslash\{i\}, C \cup\{i\}\right)\right)\right),
\end{aligned}
$$

and its relative integrated power index is

$$
R_{i}^{\prime}(v)=\frac{R_{i}(v)}{\sum_{i \in N} R_{i}(v)} .
$$

Compared to traditional Banzhaf indices $\left(B_{i}\right)$, the proposed integrated power index of voters in a voting game with a social network has the following properties.

Property 4. For any $i \in X, R_{i}=0 \Longrightarrow B_{i}=0$ and $B_{i}=0 \Longrightarrow$ $R_{i}=0$ if and only if it is an isolated node in the social network.

When $R_{i}=0$, it shows that the direct power and indirect power of the voter are both zero, which is easy to represent as $B_{i}=0$. Conversely, a voter who has no ability to influence the voting result is called a "dummy" in the traditional power measure and his/her power index is 0 , i.e., $B_{i}=0$. Although a "dummy" cannot affect the outcome directly, he/she may influence the behaviour of other voters through social interaction. Thus, his/her integrated power index is not necessarily 0 . In addition, when the "dummy" is an isolated node in the social network, he/she does not interact with other nodes and cannot generate power by influencing other nodes. Therefore, his/her recessive power index must be 0 .

Property 5. For any $i \in X, B_{i}=1 \Longrightarrow R_{i}=1$ if and only if it is an isolated node in the social network.

Similar to Property 4 , when $B_{i}=1$, it is a dictator in the traditional Banzhaf index. A dictator's power index weakens because of the influence of other individuals, and his/her recessive power index is not necessarily 1 . In contrast, when the dictator is an isolated node, the recessive power index must be 1 because he/she is not affected by other individuals.

\section{Simulation Analysis and Results}

Now we can analyze the influence of social learning on power indices. The most important dependent variables of interest are the individual attribute (professional level), social network structure, relationship closeness, and social learning efficiency. Thus, an application scenario and simulation results are described in this section.

4.1. Setup. A voting group with 9 members votes for an environmental protection project following a simple majority voting rule. Suppose the social relationships among the group can be described as a social network shown in Figure 4 . The voting weight and social information of the voters are shown in Table 1.

4.2. Results and Analysis. We analyze the power indices using the proposed model introduced in Section 3. Assuming that the social impact coefficient (social learning efficiency) $b=1$, and coefficient $\alpha^{+}=\alpha^{-}=1$, then voters' Banzhaf indices and integrated power indices can be calculated. The results are shown in Table 2, and the comparison of the integrated power indices and Banzhaf indices is shown in Figure 5.

As seen from Table 2 and Figure 5, due to the influence of social learning behaviour, the integrated power indices of 
TABLE 1: Voting weight and voters' social information.

\begin{tabular}{lccc}
\hline Voter & Voting weight & Node weight & Neighbor nodes (edge weight) \\
\hline V1 & 5 & 9 & V3(1), V9(5) \\
V2 & 2 & 8 & V3(2), V5(7), V9(6) \\
V3 & 1 & 5 & V1(1), V2(2), V8(3) \\
V4 & 4 & 3 & V2(7), V7(5), V8(9) \\
V5 & 3 & 2 & V9(4) \\
V6 & 5 & 8 & V5(5) \\
V7 & 2 & 9 & V3(3), V5(9) \\
V8 & 3 & 6 & V1(5), V2(6), V6(4) \\
V9 & 3 & 2 & \\
\hline
\end{tabular}

TABLE 2: Banzhaf indices and integrated power indices for voters.

\begin{tabular}{lccccccrrr}
\hline Voters & V1 & V2 & V3 & V4 & V5 & V6 & V7 & V8 & V9 \\
\hline$B_{i}$ & 0.4102 & 0.1523 & 0.0742 & 0.3242 & 0.2305 & 0.4102 & 0.1523 & 0.2305 & 0.2305 \\
$R_{i}$ & 0.2796 & 0.4569 & 0.2272 & 0.4499 & 0.4547 & 0.2719 & 0.2394 & 0.2819 & 0.4512 \\
$B_{i}^{\prime}$ & 0.1852 & 0.0688 & 0.0335 & 0.1464 & 0.1041 & 0.1852 & 0.0688 & 0.1041 & 0.1041 \\
$R_{i}^{\prime}$ & 0.0898 & 0.1468 & 0.0730 & 0.1445 & 0.1461 & 0.0874 & 0.0769 & 0.0906 & 0.1449 \\
\hline
\end{tabular}

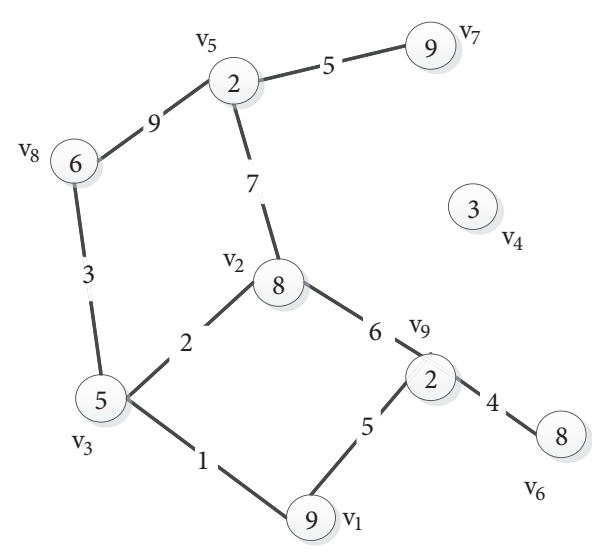

FIGURE 4: The social network in a voting group. The node $v_{i}$ represents voter $i$, the node' weight represents the knowledge professional level $k_{i}$, and the weighted edge represents the relationship closeness $\left(m_{i j}\right)$ among voters.

voters have varied changes compared to the Banzhaf indices. Voter 2 has the highest integrated power in the voting process, even though his or her Banzhaf index is only slightly higher than that of the least powerful (Banzhaf index) voter 3, whereas the integrated power indices of voter 1 and voter 6 , who originally had the highest Banzhaf index ratings, become relatively small. As shown in Figure 5, the power indices of voter 1 , voter 2 , and voter 6 changed considerably. Specifically, voter 4 is the "isolated" node in the social network, and its integrated power index varies slightly compared to the Banzhaf index.

4.2.1. The Influence of Individual Professionalism on the Power Index. Professional level is an important factor affecting a voter's social power which definitely influence the

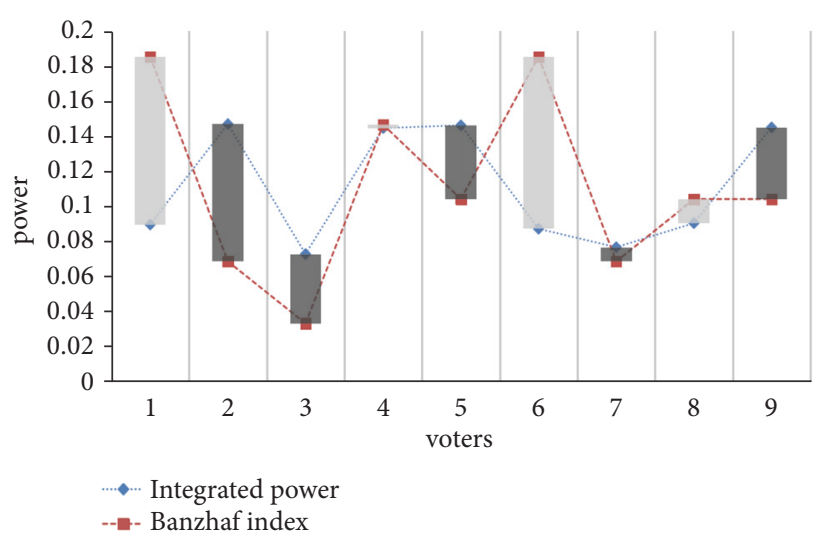

FIGURE 5: Comparison of the integrated power and Banzhaf indices.

social learning behaviour and voting power. We discuss how a voter's professional level affects power indices by analyzing the relationship between professional level $k_{i}$ and $D$ - $\operatorname{power}(i)\left(D\right.$-power $\left.(i)=R^{\prime}(i)-B^{\prime}(i)\right)$. For any voter $i \in X$, let voter $i$ have a professional level $k_{i}=1,2,3, \cdots, 9$ in turn while keeping the other parameters; the changes in power indices are shown in Figure 6.

Figure 6 shows that for any voter, with the exception of an isolated node, the greater the value of $k_{i}$ is, the higher the value of $D$ - power $(i)$ is. This result means that, in the same voting environment and conditions, the higher the voter's professional level is, the higher the integrated power index is.

Overwhelming evidences suggest that the structure of social relationship network affects the group interactions and behaviours of individuals $[15,16]$. It is necessary to test the robustness when a varied number of players are involved.

The robustness is discussed when the number of players changes based on random network structure. Figure 7 shows 


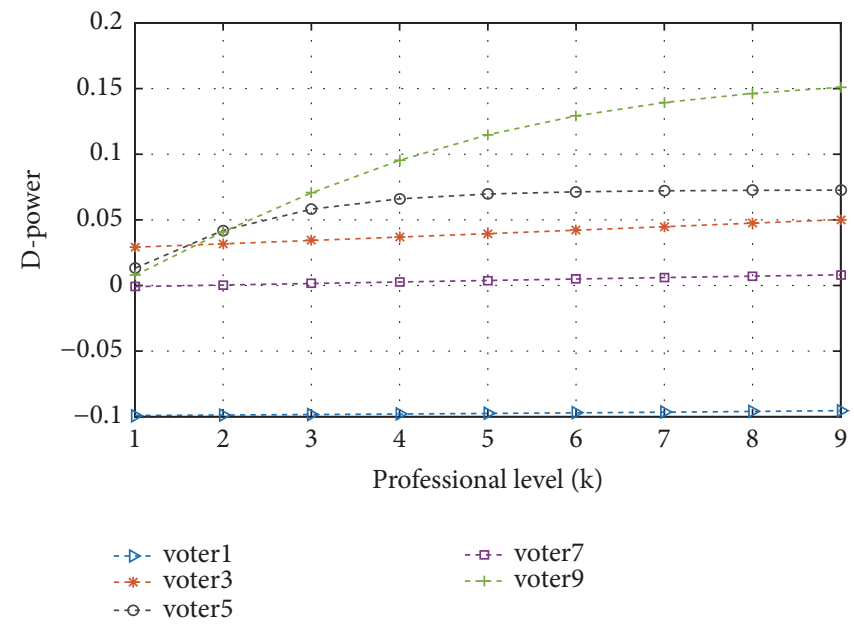

FIGURE 6: Influence of professionalism on power indices. Voters in the social network shown in Figure 4 play a repeated voting game-in which they have social learning behaviours through observing their neighbors-with voter $i$ 's professional level $k_{i}$ rising from $k_{i}=1$ to $k_{i}=9$. To make it clearer, only changes in power indices $\left(D\right.$-power $\left.(i)=R^{\prime}(i)-B^{\prime}(i)\right)$ of voter 1 , voter 3 , voter 5 , voter 7 , and voter 9 are shown. The changes in power indices improve with the professional level increasing, except for the isolated nodes.

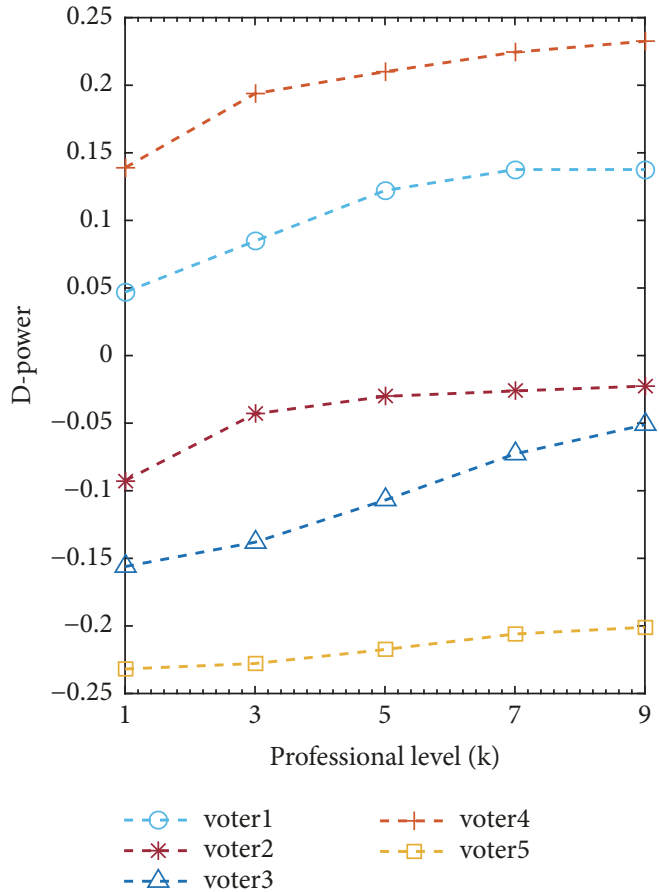

(a) The results when $\mathrm{N}=5$

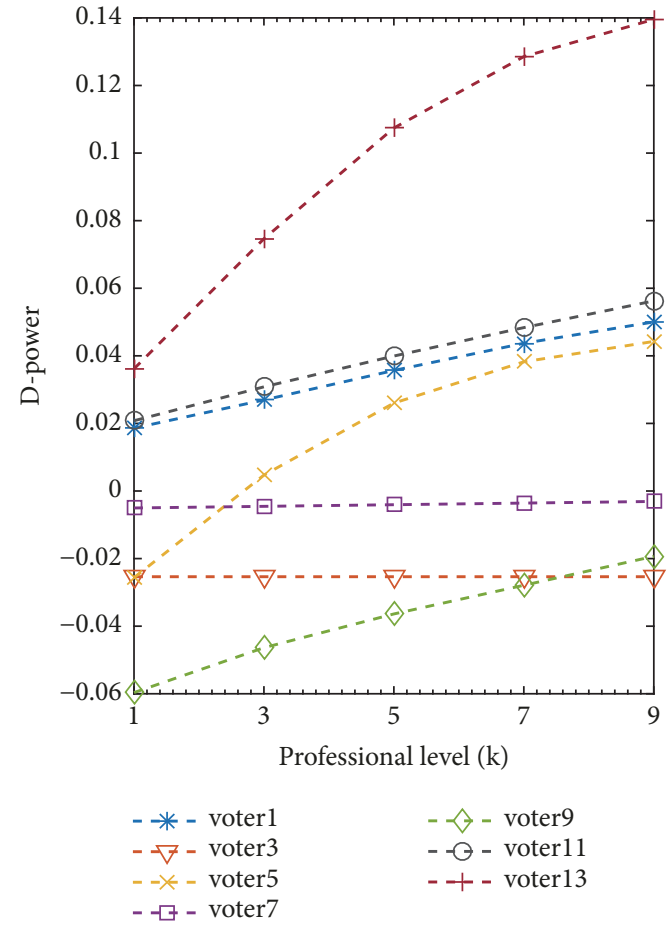

(b) The results when $\mathrm{N}=13$

FiguRE 7: Robustness of professionalism influence. Subgraph (a) shows the simulation results of 5 players (i.e., $N=5$ ) who play a repeated voting game in a social network with a $30 \%$ random connection probability, accompanied by voter $i$ s professional level $k_{i}$ rising from $k_{i}=1$ to $k_{i}=9$, while leaving other parameters unchanged. Subgraph (b) shows the results of 13 players (i.e., $N=13$ ) with the same simulation conditions. The changes in power indices $\left(D\right.$-power $\left.(i)=R^{\prime}(i)-B^{\prime}(i)\right)$ improve with the professional level increasing in both cases, except for the isolated nodes.

the simulation results when the number of players is 5 and 13. Voters play a repeated voting game in a social network with a $30 \%$ connection probability, accompanied by voter $i$ 's professional level $k_{i}$ rising from $k_{i}=1$ to $k_{i}=9$. As shown in Figure 7, regardless of the number of players, the integrated power index increases with the professional level rising, except for the isolated nodes.

The finding that the integrated power index increases with the professional level rising is in line with people's instincts: the higher the individual's professional knowledge 


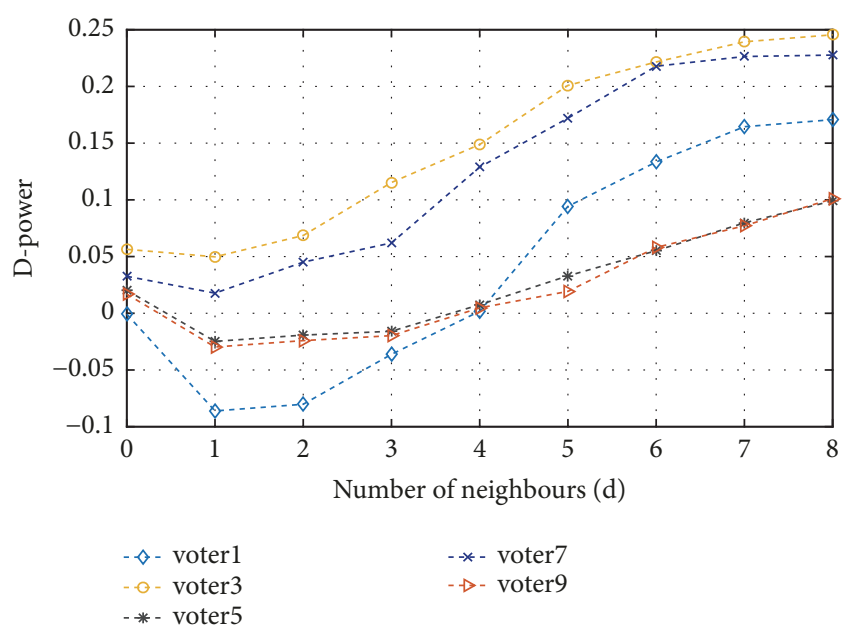

FIGURE 8: Impact of social network structure on the power index. Voters in the social network shown in Figure 4 play a repeated voting game with the number of voter $i$ 's neighbors $d_{i}$ increasing from $d_{i}=$ 0 to $d_{i}=8$ while keeping the other parameters. While the integrated power index may decrease when the number of neighbors changes from 0 to 1 , the changes in power indices $\left(D\right.$-power $\left.(i)=R^{\prime}(i)-B^{\prime}(i)\right)$ increase with the number of neighbors increasing from 1 to 8 .

or technical level is, the higher the authority or discourse power in the group is and the greater the influence over others in the interaction is; in this case, the individual will have higher integration power. Therefore, in an interactive voting game problem, if the decision-makers want to obtain more power, especially those at the low professional level, one of the important ways to improve authority is through the professional field.

4.2.2. The Influence of Social Network Structure on the Power Index. Another important parameter in which we are interested is the structure of the social network, which can be described using the network centrality of voters. To avoid interference with relationship closeness, we assume that for any pair of voters $i, j$, the relationship closeness $m_{i j}=1$. For any voter $i \in X$, let the number of voter $i$ 's neighbors changes as $d_{i}=1,2,3, \cdots, 9$ while keeping other parameters or conditions unchanged. The changes in power indices are shown in Figure 8.

As we can see from Figure 8, the value of $D$ - power(i) increases with the number of neighbors increasing, except when the voter's number of neighbors is 0 . This means that the voter may have a higher integrated power index when he/she is an isolated node in the social network, compared with having relatively fewer neighbors.

To analyze the robustness of the above finding, we discuss the influence of voter $i$ 's social structure based on the random network with a $30 \%$ connection probability. Figure 9 shows the simulation results when the number of players is either $N=5$ or $N=13$, with voter $i$ 's neighbors $d_{i}$ increasing from $d_{i}=0$ to $d_{i}=8$ while keeping the other parameters. As shown in Figure 9, the simulation results are the same with the above findings.
The analysis results show that the integrated power index of decision-makers may decrease when he/she changes from an isolated node to a nonisolated node, and then his/her integrated power will increase with the number of neighbor nodes increasing. This finding is inconsistent with people's intuitions, and the main reasons for such inconsistency are that when an individual is an isolated node in a social network, he or she is not disturbed by any other viewpoint, and the influence on the result depends entirely on his or her own judgment, i.e., direct power. When there is a social learning process, the direct power is reduced by the influence of other individuals. However, as the extensiveness of individual social relations increases, the influence on other individuals will also gradually increase, and his or her indirect power index will gradually increase. When indirect power increases to a certain extent, his or her integrated power will gradually increase. Therefore, if the extensiveness of an individual's social relations is at a relatively low level, social learning will reduce his or her power (compared with noninteractive nodes); only when the extensiveness of social relations reaches a certain high level does social learning improve his or her integrated power.

\subsubsection{The Influence of Relationship Closeness on the Power} Index. Now we examine the relationship between the closeness $\gamma_{i j}$ and power index. For any voter $i \in X$, let the relationship closeness among voter $i$ and all of his neighbor voters be equal and $m_{i j}=m_{i k}=1,2,3, \cdots, 9\left(\forall j, k \in \Gamma_{i}\right)$, while keeping other parameters or conditions unchanged; the changes in the power index are shown in Figure 10.

Figure 11 shows the simulation results when the number of players is either $N=5$ or $N=13$, within a social network constructed by a $30 \%$ connection probability, and the results shows the same that the integrated power improves with the voter's closeness accompanied by neighbors increasing.

The analysis results show that the greater the closeness is in the relationship between individual and neighbor nodes, the higher the integrated power index is. The relationship closeness is related to the social influence on the individual; the individual is more susceptible to the behaviour of the person who is closer to her or him. Therefore, in voting games, individuals with high closeness to other individuals are more likely to gain higher integration power.

4.2.4. The Influence of Social Learning Efficiency. Social learning efficiency is an important parameter that reflects the degree of interaction. We explore the impact of social learning efficiency on voting power by assuming social learning efficiency $b=1,2,3, \cdots, 9\left(j \in \Gamma_{i}\right)$ while keeping the other parameters or conditions, and the result is shown in Figure 12.

As we can see from Figure 12, with the improvement in social learning efficiency $b$, the integrated power index of voter 5 and voter 9 decreased gradually, while the integrated power index of the other individuals increased gradually. The main reason is the difference in the social network structure: Figure 4 shows that voters 5 and 9 are people with extensive relationships and high relationship closeness in the social network, and the social influence they experience is at a higher level. Thus, the improvement in social learning efficiency 


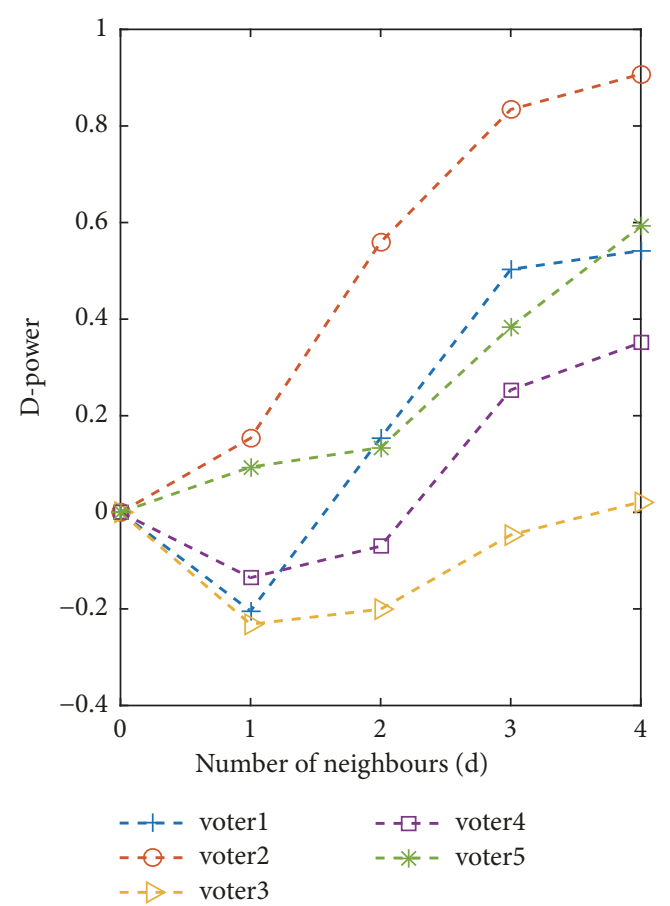

(a) The results when $\mathrm{N}=5$

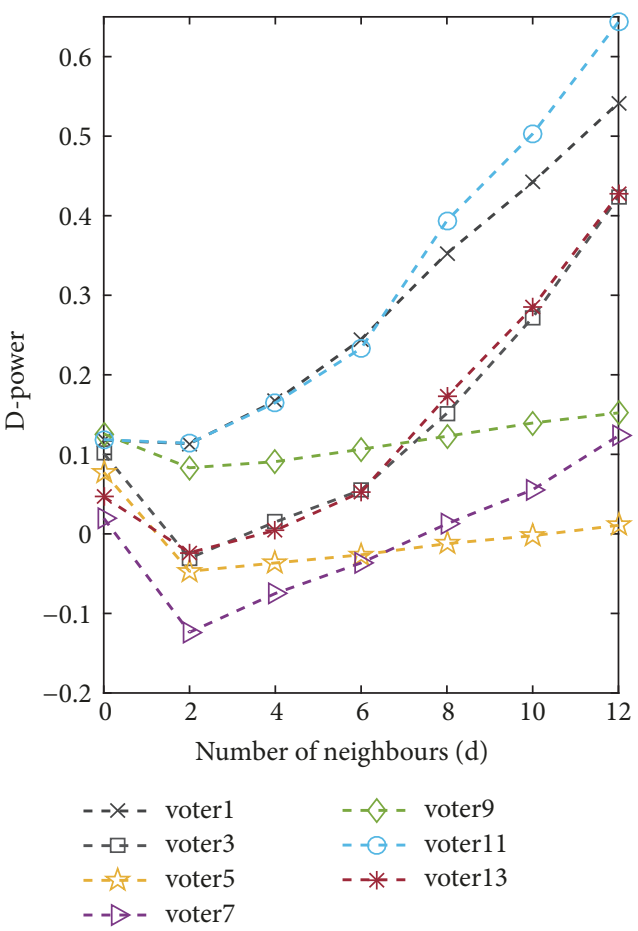

(b) The results when $\mathrm{N}=13$

FIGURE 9: Robustness of voter's neighbors influence. Subgraphs (a) and (b) show the simulation results of five players (i.e., $N=5$ ) and 13 players (i.e., $N=13$ ), respectively, in which voters play a repeated voting game in a social network with a $30 \%$ connection probability, accompanied by voter $i$ 's neighbors $d_{i}$ increasing from $d_{i}=0$ to $d_{i}=8$ while keeping the other parameters.

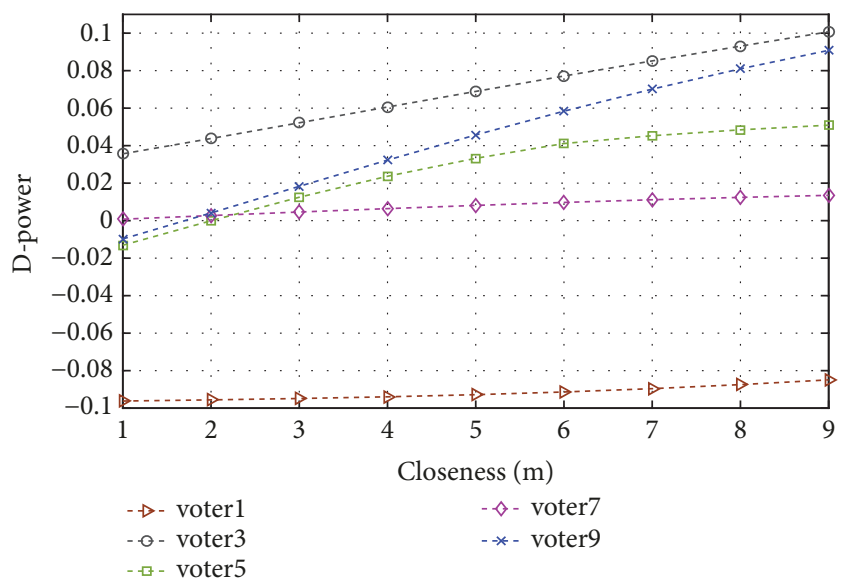

FIGURE 10: The influence of relationship closeness on the power index. Voters-within the social network shown in Figure 4-play a repeated voting game with the closeness among voter $i$ and his/her neighbor voters $m_{i j}=m_{i k}\left(\forall j, k \in \Gamma_{i}\right)$ increasing from $m_{i j}=1$ to $m_{i j}=9$ while leaving other parameters or conditions unchanged. The changes in power indices increase $\left(D\right.$-power $\left.(i)=R^{\prime}(i)-B^{\prime}(i)\right)$ with the voter's closeness with neighbors increasing.

affects them more than it does with other individuals, and the integrated power index will be relatively lower. In contrast, other individuals gained relatively high integrated power. The simulation experiment results shown in Figure 13 also present the phenomenon that a higher social learning efficiency may lead to a decrease in the integrated power index of voters with higher social impact or closeness, and the voters with lower social impact or closeness are the opposite, while both cases are possible for voters with middle social impact or closeness.

This finding shows that the effect of social learning efficiency on the integrated power index is closely related to the social network structure of an individual; for people with strong communicative competence, a higher social learning efficiency is not conducive to the promotion of their integrated power.

In brief, integrated power is directly or indirectly affected by multiple factors, such as professional knowledge level, social network structure, relationship closeness, and social learning efficiency. Only when all these factors are at higher levels can they have higher integrated power than other decision-makers. Limited social relations in a group may restrict the integrated power of an authority figure with a high level of professional knowledge. However, individuals with normal professional knowledge levels have higher power if they engage in extensive communication and garner high influence. The measurement of the integrated power index can help us find the recessive power in management and the key factors in problems, and it is beneficial for improvements in management efficiency.

\section{Conclusions}

Based on the interaction among views in a voting group, the concept of integrated power is put forward to simultaneously 


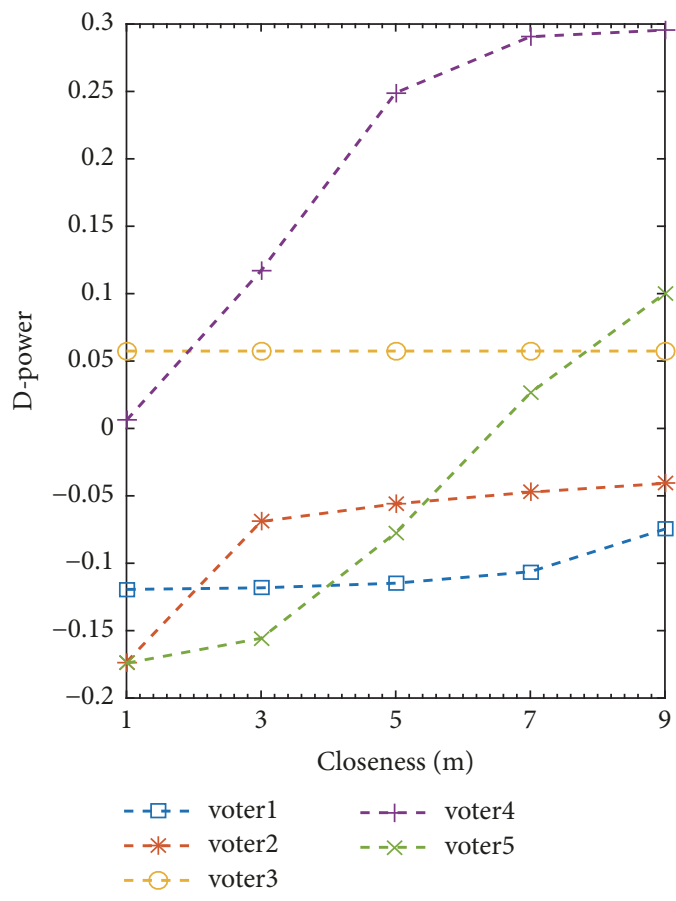

(a) The results when $\mathrm{N}=5$

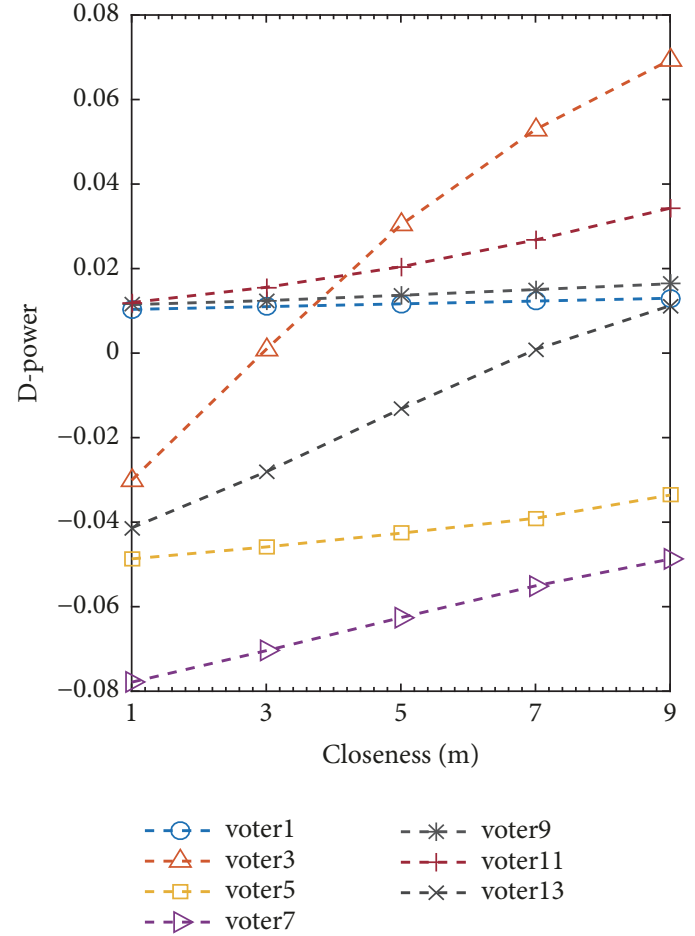

(b) The results when $\mathrm{N}=13$

Figure 11: Robustness of voter's closeness influence. Subgraphs (a) and (b) show the simulation results of five players (i.e., $N=5$ ) and 13 players (i.e., $N=13$ ), respectively. Voters play a repeated voting game in a social network with a $30 \%$ random connection probability, accompanied by voter $i$ 's closeness with his neighbors $m_{i j}=m_{i k}\left(\forall j, k \in \Gamma_{i}\right)$ increasing from $m_{i j}=1$ to $m_{i j}=9$ while keeping the other parameters.

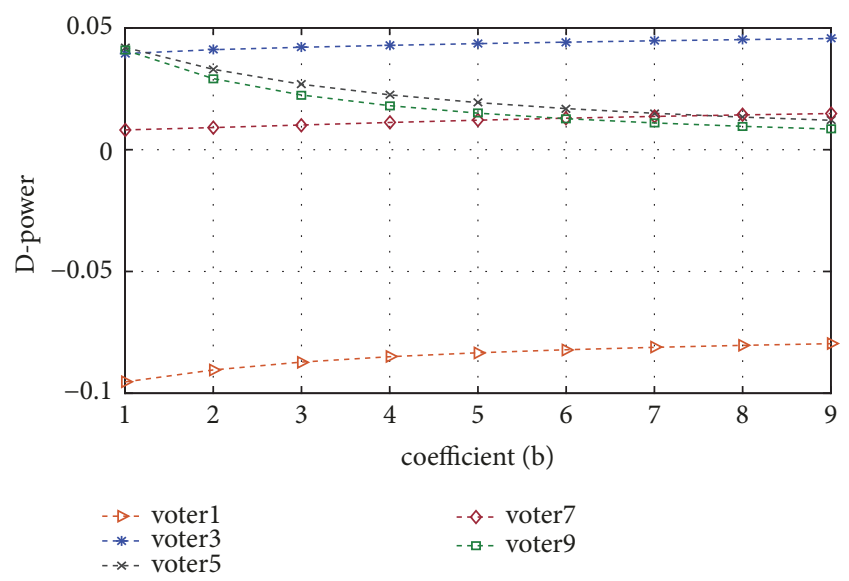

FIGURE 12: Impact of social learning efficiency on the power index. Voters-within the social network shown in Figure 4-play a repeated voting game with the social learning efficiency parameters $b$ rising from $b=1$ to $b=9$ while leaving other parameters or conditions unchanged.

measure the direct and indirect power of voters, and a method for measuring integrated power is given. We explored the influence of interaction on power indices using the theory and model of social learning. The influence of interaction on voting processes and voting results is analyzed from the perspective of voters' professional level, social network structure, relationship closeness, and social learning efficiency. We construct a measurement model of integrated power and analyze the change in integrated power relative to the Banzhaf index by numerical simulation. The results show the following:

(a) When the individual's professional level is higher and the relationship closeness with the associated decision-maker is stronger, the integrated power index is higher, and the value added is higher relative to the Banzhaf index.

(b) The integrated power index of decision-makers may decrease when he/she changes from an isolated node to a nonisolated node, and then his/her integrated power will increase with the number of neighbor nodes increasing.

(c) The influence of social learning efficiency on the integrated power index is closely related to the individual's social network structure, and for those with a strong communicative competence within the social network, higher social learning efficiency is not conducive to the promotion of integrated power; and the voters with lower social impact or closeness are the opposite, while both cases are possible for the voters with middle communicative competence. 


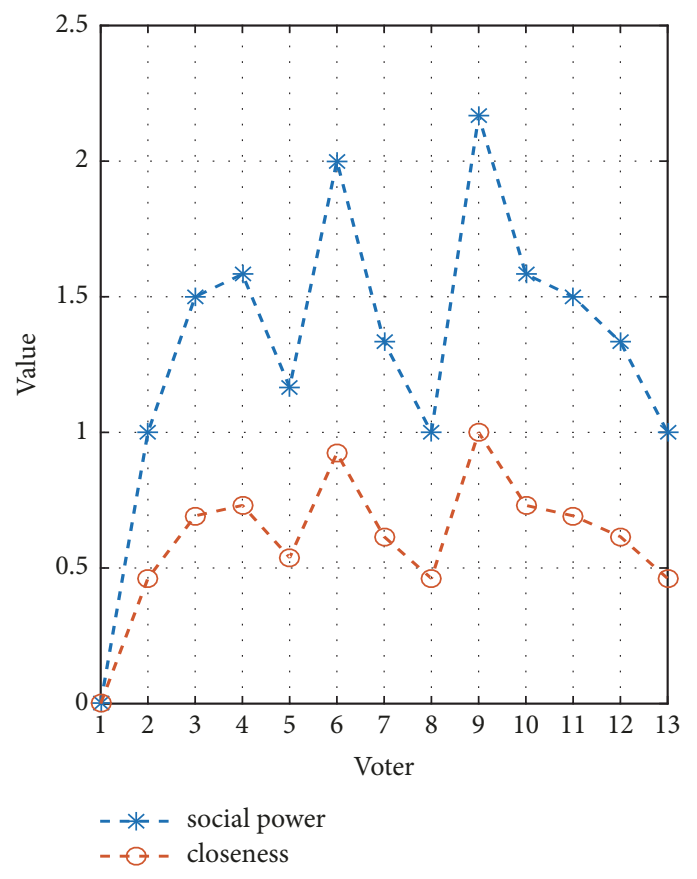

(a) Voters' social relationship structure

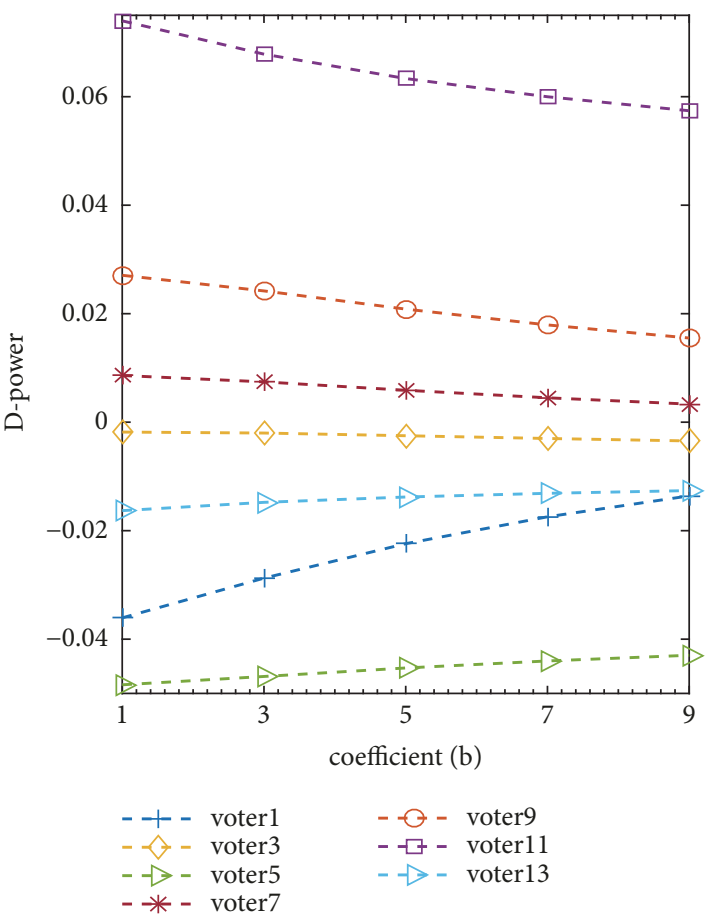

(b) Influence of learning efficiency

FIGURE 13: Impact of social learning efficiency and social relationship structure. Voters play a voting game in a social network with 13 players and a 30\% random connection probability. Subgraph (a) shows the simulation results of voters' social power and relationship closeness with neighbors. Subgraph (b) shows the simulation results of changes in power indices $\left(D\right.$-power $\left.(i)=R^{\prime}(i)-B^{\prime}(i)\right)$, in which voters play a repeated voting game with the social learning efficiency $(b)$ increasing from $b=1$ to $b=9$ while leaving other parameters unchanged. The integrated power of voters with higher social impact or closeness decreases accompanied by the increases of social learning efficiency $(b)$, and the voters with lower social impact or closeness are the opposite, while both cases are possible for the voters with middle social impact or closeness.

Compared to the previous methods of power measurement, the proposed method is no longer confined to the determination of power based on voting results but takes into account the social relationships of decision-makers and the effect that interaction has on their views. The proposed power measure includes both the direct power and the indirect power gained through a social network and can better reflect the distribution of power in the voting problem, the obligations undertaken, and the degree of contribution to the results. This measure is valuable for the identification of key decision-makers, prediction, and guidance regarding developing trends in public opinion in online networks, as well as for network recommendations, etc.

The ideas explored in this paper, however, are quite preliminary. There are many important problems in need of further research, such as the heterogeneity of the learning efficiency of each voting individual in the analysis of the social learning. The characteristics and properties of recessive power are also a key area for further research.

\section{Data Availability}

All the data used to support the findings of this study are included or described within the article.

\section{Conflicts of Interest}

The authors declare that there are no conflicts of interest regarding the publication of this paper.

\section{Acknowledgments}

This work was supported by the National Natural Science Foundation of China (Projects nos. 71471123; 71871150; 71571126), Sichuan University Innovation Spark Project (2018hhs-35), and the Fundamental Research Foundation for the Central Universities (Project no. skqy201621).

\section{References}

[1] L. S. Shapley and M. Shubik, "A method for evaluation the distribution of power in a committee system," The American Political Science Review, vol. 48, no. 3, pp. 787-792, 1988.

[2] J. F. Banzhaf, "Weighted voting doesn't work: a mathematical analysis," Rutgers Law Review, vol. 19, pp. 317-343, 1964.

[3] J. Gao, X. Yang, and D. Liu, "Uncertain Shapley value of coalitional game with application to supply chain alliance," Applied Soft Computing, vol. 56, pp. 551-556, 2017.

[4] R. R. Yager, "On using the shapley value to approximate the choquet integral in cases of uncertain arguments," IEEE 
Transactions on Fuzzy Systems, vol. 26, no. 3, pp. 1303-1310, 2018.

[5] A. Gelman, J. N. Katz, and J. Bafumi, "Standard voting power indexes do not work: an empirical analysis," British Journal of Political Science, vol. 34, no. 4, pp. 657-674, 2004.

[6] D. S. Felsenthal and M. Machover, "A priori voting power: what is it all about?" Political Studies Review, vol. 2, no. 1, pp. 1-23, 2016.

[7] S. Kaniovski and D. Leech, "A behavioral power index," Public Choice, vol. 141, no. 1-2, pp. 17-29, 2009.

[8] L. S. Shapley, "A comparison of power indices and a nonsymmetric generalization," Development, vol. 115, no. 4, pp. 957971, 1977.

[9] G. Owen and L. S. Shapley, "Optimal location of candidates in ideological space," International Journal of Game Theory, vol. 18, no. 3, pp. 339-356, 1989.

[10] C. Beisbart and L. Bovens, "A power measure analysis of Amendment 36 in Colorado," Public Choice, vol. 134, no. 3-4, pp. 231-246, 2008.

[11] L. Bovens and C. Beisbart, "Measuring voting power for dependent voters through causal models," Synthese, vol. 179, no. 1, pp. 35-56, 2011.

[12] J. Freixas, "Probabilistic power indices for voting rules with abstention," Mathematical Social Sciences, vol. 64, no. 1, pp. 8999, 2012.

[13] S. Courtin and B. Tchantcho, "A note on the ordinal equivalence of power indices in games with coalition structure," Theory and Decision. An International Journal for Multidisciplinary Advances in Decision Science, vol. 78, no. 4, pp. 617-628, 2015.

[14] S. Courtin, Z. Nganmeni, and B. Tchantcho, "The shapleyshubik power index for dichotomous multi-type games," Theory and Decision, vol. 81, no. 3, pp. 1-14, 2016.

[15] M. Perc, J. Gómez-Gardeñes, A. Szolnoki, L. M. Floría, and Y. Moreno, "Evolutionary dynamics of group interactions on structured populations: a review," Journal of the Royal Society Interface, vol. 10, no. 80, Article ID 20120997, 2013.

[16] M. Perc, J. J. Jordan, D. G. Rand, Z. Wang, S. Boccaletti, and A. Szolnoki, "Statistical physics of human cooperation," Physics Reports, vol. 687, pp. 1-51, 2017.

[17] G. Cao, Y. Shi, and Q. Li, "Structure characteristics of the international stock market complex network in the perspective of whole and part," Discrete Dynamics in Nature and Society, vol. 1, pp. 1-11, 2017.

[18] A. E. Sokhey and S. D. McClurg, "Social networks and correct voting," Journal of Politics, vol. 74, no. 3, pp. 751-764, 2012.

[19] O. Lesser, L. Naamani-Dery, M. Kalech, and Y. Elovici, "Group decision support for leisure activities using voting and social networks," Group Decision and Negotiation, vol. 26, no. 3, pp. 473-494, 2017.

[20] E. Katz and P. F. Lazarsfeld, Personal Influence: The Part Played by People in The Flow of Mass Communications, Transaction Publishers, Piscataway, NJ, USA, 2006.

[21] R. V. D. Brink, A. Rusinowska, and F. Steffen, "Measuring power and satisfaction in societies with opinion leaders: dictator and opinion leader properties," Homo Oeconomicus, vol. 28, no. 1-2, pp. 161-185, 2011.

[22] R. van den Brink, A. Rusinowska, and F. Steffen, "Measuring power and satisfaction in societies with opinion leaders: an axiomatization," Social Choice and Welfare, vol. 41, no. 3, pp. 671-683, 2013.

[23] R. van den Brink, A. Rusinowska, and F. Steffen, "Satisfaction in societies with opinion leaders and mediators: properties and an axiomatization," Computer Science and Game Theory, 2014.
[24] J. Feng and Z. Yao, "Consumer-generated reviews based on social learning theory: implications for purchase decision," Chinese Journal of Management Science, vol. 24, no. 9, pp. 106$114,2016$.

[25] B. Kittel, G. Kanitsar, and S. Traub, "Knowledge, power, and selfinterest," Journal of Public Economics, vol. 150, pp. 39-52, 2017.

[26] C. Guo, R.-L. Shi, and M. Jin, "Group decision opinion evolution and simulation based on community and individual influence power," Journal of Intelligent \& Fuzzy Systems, vol. 33, no. 5, pp. 2667-2676, 2017.

[27] J. Wu, F. Chiclana, H. Fujita, and E. Herrera-Viedma, "A visual interaction consensus model for social network group decision making with trust propagation," Knowledge-Based Systems, vol. 122, pp. 39-50, 2017.

[28] J. Wu, L. Dai, F. Chiclana, H. Fujita, and E. Herrera-Viedma, "A minimum adjustment cost feedback mechanism based consensus model for group decision making under social network with distributed linguistic trust," Information Fusion, vol. 41, pp. 232 242, 2018.

[29] Y. T. Mohmand and A. Wang, "Weighted complex network analysis of pakistan highways," Discrete Dynamics in Nature \& Society, vol. 4, pp. 1-5, 2013.

[30] L. C. Freeman, "Centrality in social networks conceptual clarification," Social Networks, vol. 1, no. 3, pp. 215-239, 1978.

[31] S. B. Seidman, "Network structure and minimum degree," Social Networks, vol. 5, no. 3, pp. 269-287, 1983.

[32] F. Morone and H. A. Makse, "Influence maximization in complex networks through optimal percolation," Nature, vol. 524, no. 7563, pp. 65-68, 2015.

[33] C. Simon de Blas, J. Simon Martin, and D. Gomez Gonzalez, "Combined social networks and data envelopment analysis for ranking," European Journal of Operational Research, vol. 266, no. 3, pp. 990-999, 2018.

[34] A. Bandura, Social Learning Theory, vol. 1, ReCAPP, Scotts Valley, Calif, USA, 2000.

[35] P. Kristjanson, B. Harvey, M. Van Epp, and P. K. Thornton, "Social learning and sustainable development," Nature Climate Change, vol. 4, no. 1, pp. 5-7, 2014.

[36] L. Qiu and A. B. Whinston, "Pricing strategies under behavioral observational learning in social networks," Production Engineering Research and Development, vol. 26, no. 7, pp. 1249-1267, 2017.

[37] S. Kurz, "Measuring Voting Power in Convex Policy Spaces," Economies, vol. 2, no. 1, pp. 45-77, 2014.

[38] P. Hu and S. Z. Huang, "Analysis and measuring model of voting individuals power on weighted majority rules," Journal of management science in China, vol. 4, no. 6, pp. 66-70, 2001.

[39] T. N. Dinh, Y. Xuan, M. T. Thai, P. M. Pardalos, and T. Znati, “On new approaches of assessing network vulnerability: hardness and approximation," IEEE/ACM Transactions on Networking, vol. 20, no. 2, pp. 609-619, 2012.

[40] Y. Shen, N. P. Nguyen, Y. Xuan, and M. T. Thai, "On the discovery of critical links and nodes for assessing network vulnerability," IEEE/ACM Transactions on Networking, vol. 21, no. 3, pp. 963-973, 2013.

[41] W. Asif, H. K. Qureshi, M. Rajarajan, and M. Lestas, "Combined banzhaf \& diversity index (CBDI) for critical node detection," Journal of Network and Computer Applications, vol. 64, pp. 7688, 2016.

[42] H. C. Kelman, "Interests, relationships, identities: Three central issues for individuals and groups in negotiating their social environment," Annual Review of Psychology, vol. 57, no. 1, pp. $1-26,2006$. 
[43] W. P. Erchul and B. H. Raven, "Social power in school consultation: a contemporary view of French and Raven's bases of power model," Journal of School Psychology, vol. 35, no. 2, pp. 137-171, 1997.

[44] M. Jalili, "Social power and opinion formation in complex networks," Physica A: Statistical Mechanics and Its Applications, vol. 392, no. 4, pp. 959-966, 2013. 


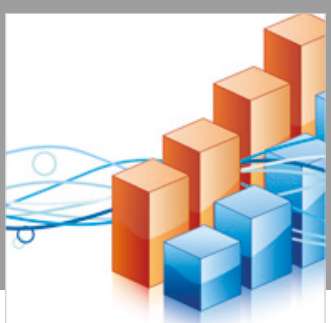

Advances in

Operations Research

\section{-n-m}
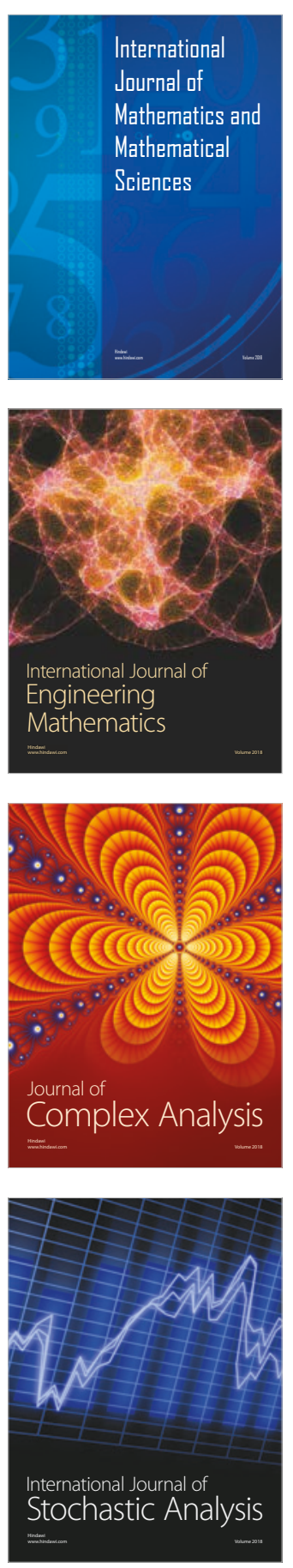
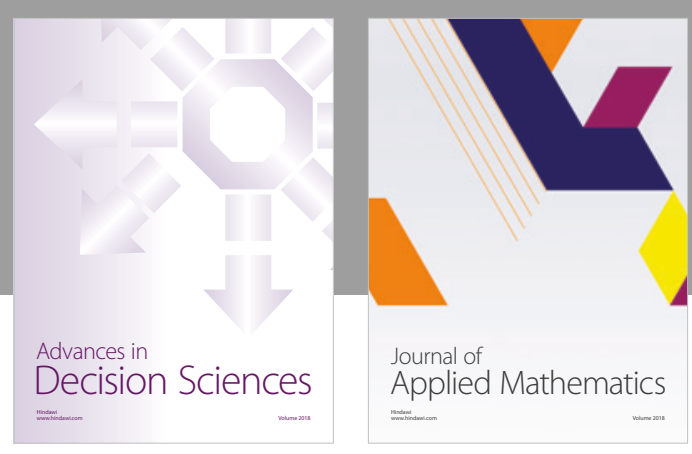

Journal of

Applied Mathematics
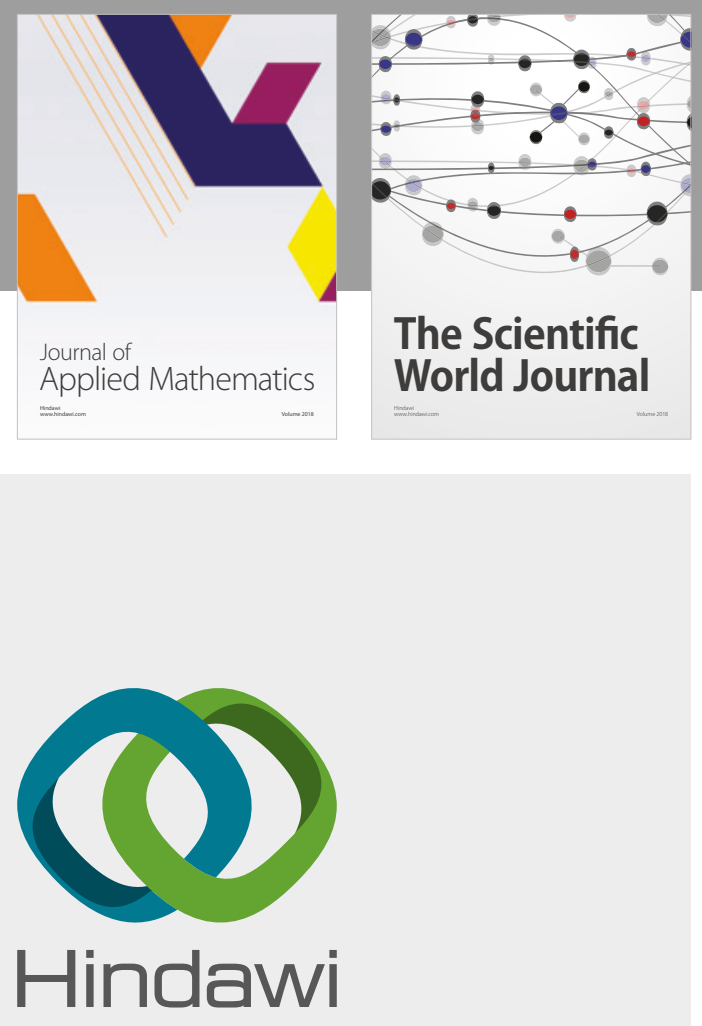

Submit your manuscripts at

www.hindawi.com

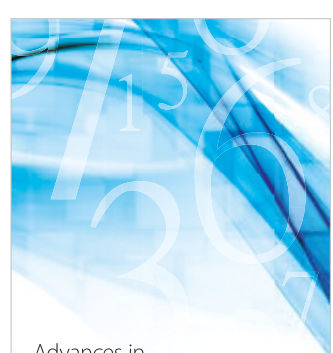

Advances in
Numerical Analysis
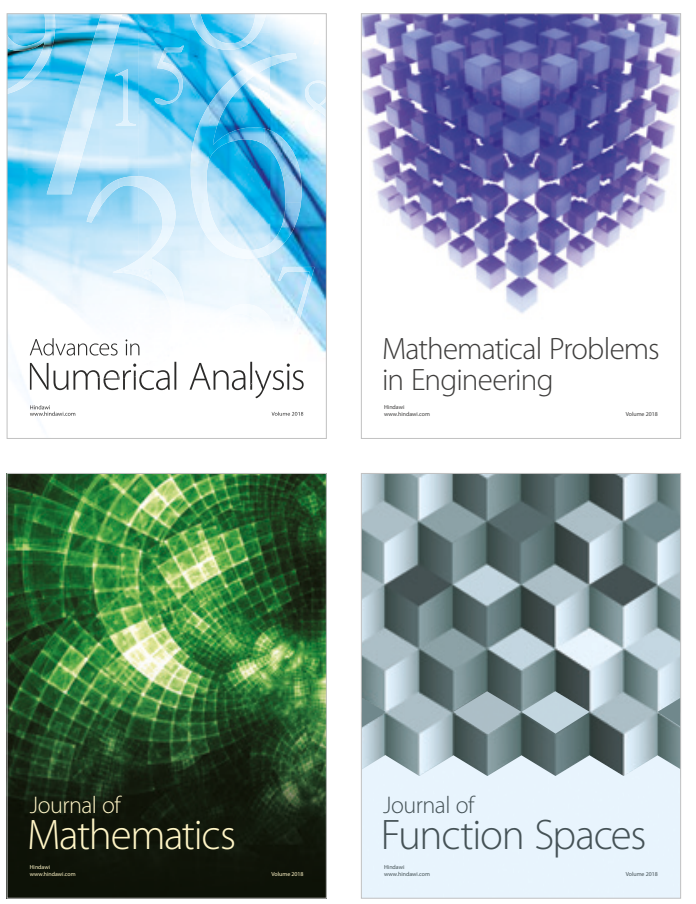

Mathematical Problems in Engineering

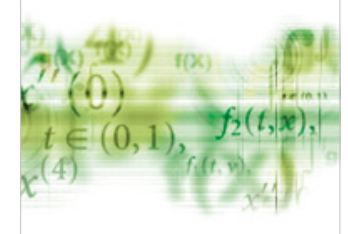

International Journal of

Differential Equations

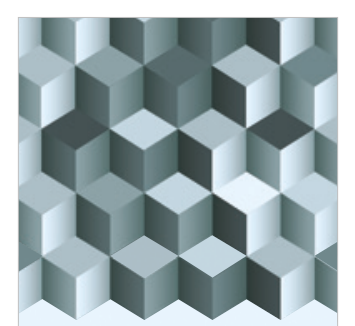

Journal of

Function Spaces
The Scientific

World Journal

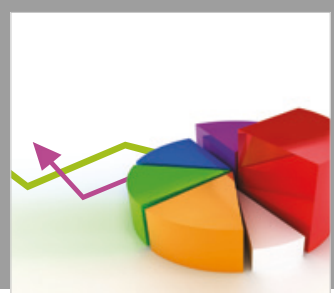

Journal of

Probability and Statistics
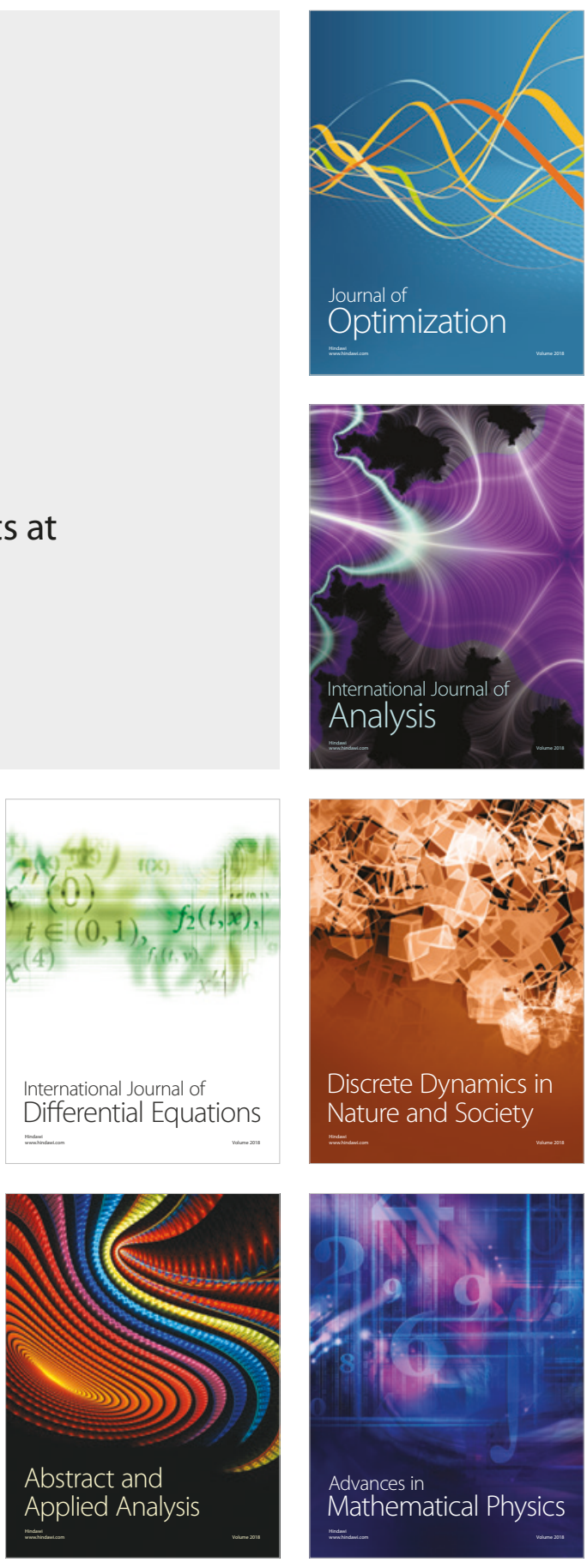